\title{
Dynamic Quantum Bernoulli Random Walks
}

\author{
Nadine Guillotin-Plantard * $\quad$ René Schott ${ }^{\dagger}$
}

Key words: Bernoulli random variables, dynamic random walks, dual group theory, dynamical systems, limit theorem, transition kernel, recurrence, transience .

AMS Subject classification: 60G50, 60J15.

\begin{abstract}
Quantum Bernoulli random walks can be realized as random walks on the dual of $S U(2)$. We use this realization in order to study a model of dynamic quantum Bernoulli random walk with time dependent transitions. For the corresponding dynamic random walk on the dual of $S U(2)$, we prove several limit theorems (local limit theorem, central limit theorem, law of large numbers, large deviations principle). In addition, we characterize a large class of transient dynamic random walks.
\end{abstract}

\section{Introduction}

Systems of commuting spins can be viewed as a non-commutative analogue of classical Bernoulli random walks. More precisely, they contain three orthogonal Bernoulli random walks. The non-commutativity comes from the fact

\footnotetext{
*Université Claude Bernard-Lyon 1, Institut Camille Jordan, 43 bld du 11 novembre 1918, 69622 Villeurbanne, France, e-mail: nadine.guillotin@univ-lyon1.fr

†IECN and LORIA, Université Henri Poincaré-Nancy 1, 54506 Vandoeuvre-lès-Nancy, France, e-mail: schott@loria.fr
} 
that these three random walks do not commute. This triple of random walks is called by P. Biane a quantum Bernoulli random walk (in short QBRW ). P. Biane has proven many fundamental properties of these random walks (see $[2,3,4,5])$. In particular he has shown that QBRWs can be realized as random walks on the dual of $S U(2)$. These random walks have been extensively studied by B. Roynette et al. [19, 9], L. Gallardo et al. [10, 11]. A theory of dynamic random walks on the euclidean space (i.e. the transition probabilities are in some sense time dependent) has been developed over the last decade by N. Guillotin [12, 13, 14] and then extended to Heisenberg groups in [17]. This theory has also been applied in computer science [15, 8] and mathematical finance [18] (see also [16]). The purpose of this paper is to introduce the notion of dynamic quantum Bernoulli random walks (in short DQBRW) and to investigate their realisations as dynamic random walks on the dual of $S U(2)$. We prove a local limit theorem, a central limit theorem, a law of large numbers, a large deviations principle and characterize a large class of transient dynamic random walks. This paper can be viewed as another (small) step in extending the theory of dynamic random walks to non-commutative algebraic structures.

The organization is as follows:

\section{Quantum Bernoulli random walks}

The presentation of this introductory material follows [3].

Let $M_{2}(\mathbb{C})$ be the set of $2 \times 2$ matrices with complex coefficients. The set of $2 \times 2$ self-adjoint matrices forms a four dimensional real vector subspace of $M_{2}(\mathbb{C})$. A convenient basis is given by the following matrices

$$
I=\left(\begin{array}{ll}
1 & 0 \\
0 & 1
\end{array}\right) \quad \sigma_{x}=\left(\begin{array}{cc}
1 & 0 \\
0 & -1
\end{array}\right) \quad \sigma_{y}=\left(\begin{array}{cc}
0 & 1 \\
1 & 0
\end{array}\right) \quad \sigma_{z}=\left(\begin{array}{cc}
0 & -\mathrm{i} \\
\mathrm{i} & 0
\end{array}\right)
$$

$\sigma_{x}, \sigma_{y}, \sigma_{z}$ are the traditional Pauli matrices, they satisfy the commutation relations: $\left[\sigma_{x}, \sigma_{y}\right]=2 i \sigma_{z}$, and those obtained by cyclic permutations of $\sigma_{x}$, $\sigma_{y}, \sigma_{z}$. Every unitary matrix $\mathrm{U}$ gives rise to a ${ }^{*}$-automorphism of $M_{2}(\mathbb{C})$

$$
\tau_{U}: M \rightarrow U M U^{*}
$$

These automorphisms leave the hermitian product $\langle M, N\rangle=\frac{1}{2} \operatorname{tr}\left(M N^{*}\right)$ invariant and $M_{2}(\mathbb{C})$ decomposes into two invariant subspaces for this action: 
$\mathbb{C} I$ and the subspace generated by $\sigma_{x}, \sigma_{y}, \sigma_{z} \cdot \tau_{U}$ acts by rotation on $\mathbb{R} \sigma_{x}+$ $\mathbb{R} \sigma_{y}+\mathbb{R} \sigma_{z}$.

A state on $M_{2}(\mathbb{C})$ is given by a density matrix which we will suppose to be of the form

$$
\left(\begin{array}{ll}
\mathrm{p} & 0 \\
0 & \mathrm{q}
\end{array}\right)
$$

with $0<p \leq 1, p+q=1$, since this can be achieved by conjugation by a unitary matrix. We denote by $\rho_{p}$ the state given by this density matrix.

Given a self adjoint element of $M_{2}(\mathbb{C})$, we can compute its law in the state $\rho_{p}$ : let $A=\lambda \sigma_{x}+\mu \sigma_{y}+\nu \sigma_{z}$, and $\xi=\left(\lambda^{2}+\mu^{2}+\nu^{2}\right)^{1 / 2}$, the spectrum of $A$ is $\{-\xi,+\xi\}$, its expectation in the state $\rho_{p}$ is $\lambda(p-q)$ and its law is given by

$$
\begin{aligned}
\mathbb{P}(A=\xi) & =\frac{1}{2}\left[\frac{\lambda(p-q)}{\xi}+1\right] \\
\mathbb{P}(A=-\xi) & =\frac{1}{2}\left[\frac{\lambda(q-p)}{\xi}+1\right]
\end{aligned}
$$

In particular, in the state $\rho_{p}, \sigma_{y}$ and $\sigma_{z}$ are symmetric Bernoulli random variables, whereas $\sigma_{x}$ takes 1 with probability $p$ and -1 with probability $q$. Let $M_{1}, M_{2}, \ldots, M_{n}, \ldots$, be infinitely many copies of $M_{2}(\mathbb{C})$. For each $p \in$ ]0,1], we consider the algebra

$$
\mathcal{M}_{p}=M_{1} \otimes \ldots \otimes M_{k} \otimes \ldots
$$

where the product is taken with respect to the product state

$$
w_{p}=\rho_{p} \otimes \ldots \rho_{p} \otimes \ldots
$$

Consider the elements $x_{k}, y_{k}, z_{k}$ of $\mathcal{M}_{p}$ given by:

$$
\begin{aligned}
x_{k} & =I \otimes \ldots \otimes I \otimes \sigma_{x} \otimes I \otimes I \ldots \\
y_{k} & =I \otimes \ldots \otimes I \otimes \sigma_{y} \otimes I \otimes I \ldots \\
z_{k} & =I \otimes \ldots \otimes I \otimes \sigma_{z} \otimes I \otimes I \ldots
\end{aligned}
$$

where each $\sigma$. appears at the $k^{t h}$ place. Define for $k \geq 1$,

$$
X_{k}=\sum_{i=1}^{k} x_{i}, \quad Y_{k}=\sum_{i=1}^{k} y_{i}, \quad Z_{k}=\sum_{i=1}^{k} z_{i}
$$

and $X_{0}=Y_{0}=Z_{0}=0$.

The triple $\left(X_{n}, Y_{n}, Z_{n}\right)_{n \in \mathbb{N}}$ is called a quantum random walk. 


\section{The dual of $S U(2)$}

The presentation in this section follows [19].

$S U(2)$ is the group of matrices

$$
g=\left(\begin{array}{cc}
a & b \\
-\bar{b} & \bar{a}
\end{array}\right)
$$

where $a$ and $b$ are complex numbers such that $|a|^{2}+|b|^{2}=1$. It is a compact Lie group. For each $x \in \mathbb{N}$, let $H_{x}$ be the vector space on $\mathbb{C}$ of polynomials of degree less than or equal to $x$. If $g$ is as indicated above and if $p \in H_{x}$, let

$$
\left[\pi_{x}(g) p\right](z)=(b z+\bar{a})^{x} p\left(\frac{a z-\bar{b}}{b z+\bar{a}}\right), z \in \mathbb{C} .
$$

In this way we define an irreducible, continuous representation of dimension $x+1$ from $S U(2)$ into $H_{x}$. It is known that as $x$ goes trough $\mathbb{N}$ then the $\pi_{x}$ produce (up to equivalence) the full list of unitary irreducible representations of $S U(2)$. Therefore $\mathbb{N}$ can be identified with the dual group of $S U(2)$.

The character of $\pi_{x}$ is given by the formula

$$
\xi_{x}(g)=\frac{1}{x+1} \operatorname{tr}\left(\pi_{x}(g)\right)=\frac{\sin [(x+1) \theta]}{(x+1) \sin \theta}
$$

where $e^{i \theta}, e^{-i \theta}$ are the eigenvalues of the matrix $g \in S U(2) . \quad \xi_{x}$ obeys $\xi_{x}\left(g_{1} g_{2}\right)=\xi_{x}\left(g_{1}\right) \xi_{x}\left(g_{2}\right)$ for all $g_{1} \in S U(2), g_{2} \in S U(2)$.

Notation: $\phi(\theta)$ will stand for $\phi(M)$ where

$$
M=\left(\begin{array}{cc}
e^{i \theta} & 0 \\
0 & e^{-i \theta}
\end{array}\right)
$$

For the characters we get the orthogonality relations with respect to the measure

$$
d \lambda(\theta)=\frac{2}{\pi} \sin ^{2} \theta d \theta
$$

More precisely, for $x \in \mathbb{N}$ and $y \in \mathbb{N}$,

$$
\int_{0}^{\pi} \xi_{x}(\theta) \xi_{y}(\theta) d \lambda(\theta)= \begin{cases}0 & \text { if } x \neq y \\ (x+1)^{-2} & \text { if } x=y\end{cases}
$$

Finally, we recall Clebsch-Gordan's formula for $S U(2)$.

If $x \leq y$ are in $\mathbb{N}$, then the tensorial product of the representations $\pi_{x}$ and 
$\pi_{y}$ splits into the direct sum of a finite number of irreducible representations as follows:

$$
\pi_{x} \otimes \pi_{y}=\pi_{y-x} \oplus \pi_{y-x+2} \oplus \ldots \oplus \pi_{y+x-2} \oplus \pi_{y+x}
$$

This formula leads to the character multiplication formula:

$\xi_{x} \xi_{y}=\frac{|x-y|+1}{(x+1)(y+1)} \xi_{|x-y|}+\frac{|x-y|+3}{(x+1)(y+1)} \xi_{|x-y|+2}+\ldots+\frac{x+y+1}{(x+1)(y+1)} \xi_{x+y}$ where $x \in \mathbb{N}, y \in \mathbb{N}$ and the integers run from $|x-y|$ to $x+y$ by jumps of length two.

Now let $\mathcal{P}(\mathbb{N})$ be the set of probability measures $\mu=\sum_{x \in \mathbb{N}} a_{x} \delta_{x}$ on $\mathbb{N}$, where $\delta_{x}$ is the Dirac measure at point $x$ and $a_{x}$ are coefficients which are non negative and $\sum_{x>0} a_{x}=1$. We define a generalized convolution denoted $\star$ as follows:

$\delta_{x} \star \delta_{y}=\frac{|x-y|+1}{(x+1)(y+1)} \delta_{|x-y|}+\frac{|x-y|+3}{(x+1)(y+1)} \delta_{|x-y|+2}+\ldots+\frac{x+y+1}{(x+1)(y+1)} \delta_{x+y}$

and more generally, if $\mu, \nu$ are in $\mathcal{P}(\mathbb{N})$ :

$$
\mu \star \nu=\left(\sum_{x \geq 0} a_{x} \delta_{x}\right) \star\left(\sum_{y \geq 0} b_{y} \delta_{y}\right)=\sum_{x, y \geq 0} a_{x} b_{y} \delta_{x} \star \delta_{y}
$$

and we denote by $\mu^{n}$ the probability measure $\mu \star \mu \star \ldots \star \mu$ ( $n$ times).

The (generalized) Fourier transform of $\mu=\sum_{x \in \mathbb{N}} a_{x} \delta_{x} \in \mathcal{P}(\mathbb{N})$ is the function $\hat{\mu}$ defined on $[0, \pi]$ by

$$
\hat{\mu}(\theta)=\sum_{x \geq 0} a_{x} \xi_{x}(\theta)=\sum_{x \geq 0} \frac{a_{x} \sin [(x+1) \theta]}{(x+1) \sin \theta} .
$$

The coefficient $a_{x}$ of the measure $\mu$ can be obtained from $\hat{\mu}$ by the following formula

$$
\begin{aligned}
a_{x} & =(x+1)^{2} \int_{0}^{\pi} \hat{\mu}(\theta) \xi_{x}(\theta) d \lambda(\theta) \\
& =\frac{2(x+1)}{\pi} \int_{0}^{\pi} \hat{\mu}(\theta) \sin [(x+1) \theta] \sin \theta d \theta .
\end{aligned}
$$

In particular $\hat{\delta_{x}}=\xi_{x}$ and $\left(\widehat{\delta_{x} \star} \delta_{y}\right)=\hat{\delta_{x}} \hat{\delta_{y}}$. More generally,

$$
(\widehat{\mu \star \nu})=\widehat{\mu} \widehat{\nu} \text {. }
$$

The (generalized) Laplace transform of $\mu=\sum_{x \in \mathbb{N}} a_{x} \delta_{x} \in \mathcal{P}(\mathbb{N})$ is the function $\tilde{\mu}$ defined on $\mathbb{R}^{+}$by

$$
\tilde{\mu}(t)=\sum_{x \geq 0} \frac{a_{x} \sinh [(x+1) t]}{(x+1) \sinh (t)} .
$$


For every $x \geq 0$, we denote by $\psi_{x}$ the function

$$
t \rightarrow \frac{\sinh [(x+1) t]}{(x+1) \sinh (t)}
$$

The Laplace transform of the Dirac measure at $x \in \mathbb{N}$ is then $\tilde{\delta}_{x}=\psi_{x}$. The Clebsch-Gordan's formula can be extended as follows:

$\psi_{x} \psi_{y}=\frac{|x-y|+1}{(x+1)(y+1)} \psi_{|x-y|}+\frac{|x-y|+3}{(x+1)(y+1)} \psi_{|x-y|+2}+\ldots+\frac{x+y+1}{(x+1)(y+1)} \psi_{x+y}$.

From the definition of the generalized convolution, relation (7) and the fact that $\tilde{\delta_{x}}=\psi_{x}$, we obtain the formula

$$
(\widetilde{\mu \star \nu})=\tilde{\mu} \tilde{\nu}
$$

Let $\mu \in \mathcal{P}(\mathbb{N})$. For each $x \in \mathbb{N}$ and for each subset $A$ of $\mathbb{N}$, we consider the transition kernel from $\mathbb{N}$ to $\mathbb{N}$ :

$$
P(x, A)=\delta_{x} \star \mu(A) .
$$

Let $\left(\Omega=\mathbb{N}^{\mathbb{N}},\left(X_{n}\right)_{n \geq 0},\left(P_{x}\right)_{x \in \mathbb{N}}\right)$ be the canonical Markov chain associated with the kernel $P$. This chain will be called the random walk of law $\mu$ on $\mathbb{N}$. In other words: the probability $P(x, y)$ to be in the state $y$ at time $n+1$ when departing from state $x$ at time $n$, is equal to the coefficient on $\delta_{y}$ of the probability measure $\delta_{x} \star \mu$.

\section{Quantum Bernoulli random walks as ran- dom walks on the dual of $S U(2)$}

P. Biane [3] has proved that QBRW can be related to a random walk of the dual of $S U(2)$. In this section we outline his observation.

Let $\mathcal{A}$ be the von Neuman algebra of $S U(2)$. This is the von Neuman algebra of operators on $L^{2}(S U(2))$ generated by the left translation operators $\lambda_{g}: \lambda_{g}(f(h))=f\left(g^{-1} h\right)$ (see [7]). It is thought of as an algebra of functions on a non-commutative lattice: the dual of $S U(2)$.

Let $\frac{1}{\sqrt{2}} X, \frac{1}{\sqrt{2}} Y, \frac{1}{\sqrt{2}} Z$ be an orthonormal basis of the Lie algebra of right invariant vector fields on $S U(2)$ (with respect to the Killing inner product). Then $i X, i Y, i Z$ induce self-adjoint operators on $L^{2}(S U(2))$ which are affiliated to 
$\mathcal{A}$. The spectrum of these operators is $\mathbb{Z}$, so that one can see them as coordinate functions on the dual of $S U(2)$. The algebra $\mathcal{A}$ is endowed with a structure of cocommutative bialgebra by the morphism of algebras $m: \mathcal{A} \rightarrow \mathcal{A}$ determined by $\lambda_{g} \rightarrow \lambda_{g} \otimes \lambda_{g}$. In order to define a non commutative random walk on $\mathcal{A}$, we let $\theta$ be an irreducible 2-dimensional representation of $S U(2)$ (see Section 3). We get a quantum Markov chain on $\mathcal{A}$ whose generator $Q$ is given by

$$
Q_{p}\left(\lambda_{g}\right)=\rho_{p}(\theta(g)) \lambda_{g}
$$

Let $\mathcal{N}$ be a von Neuman algebra and $\tau$ a morphism: $\mathcal{A} \rightarrow \mathcal{A} \otimes \mathcal{N}$. Let $\mathcal{W}=\mathcal{A} \otimes \mathcal{N} \ldots \otimes \mathcal{N} \otimes \ldots$, the tensor product being taken with respect to the product space $w=\rho \otimes \ldots \otimes \rho \otimes \ldots$

We define $T: \mathcal{W} \rightarrow \mathcal{W}$ by $T=\tau \otimes s$ where $s: \mathcal{N}^{[1, \infty[} \rightarrow \mathcal{N}^{[2, \infty[}$ is the right shift. We construct morphisms $j_{n}: \mathcal{A} \rightarrow \mathcal{W}$ by putting $j_{n}=T^{n} \circ i$ where $i: \mathcal{A} \rightarrow \mathcal{W}$ is the canonical injection.

The family of morphisms $\left(j_{n}\right)$ forms a non-commutative process in the sense of Accardi, Frigerio, Lewis [1] and the triple $\left(j_{n}(i X), j_{n}(i Y), j_{n}(i Z)\right)$ forms a quantum Bernoulli random walk.

\section{Dynamic random walks on the dual of $S U(2)$}

\subsection{Definition}

In Section 3 we have considered the set $\mathcal{P}(\mathbb{N})$ of probability measures $\mu=$ $\sum_{x \in \mathbb{N}} a_{x} \delta_{x}$ on $\mathbb{N}$, where $\delta_{x}$ is the Dirac measure at point $x$ and $a_{x}$ are coefficients which are non negative and $\sum_{x \in \mathbb{N}} a_{x}=1$.

We consider now a sequence of probability measures $\left(\mu_{i}\right)_{i \geq 1}$ where $\mu_{i}=$ $\sum_{x \in \mathbb{N}} a_{x}^{(i)} \delta_{x}$ on $\mathbb{N}$, where $a_{x}^{(i)}$ are coefficients which depend on $i$ and $x$, are non negative and for every $i \geq 1, \sum_{x \in \mathbb{N}} a_{x}^{(i)}=1$.

For every $i \geq 1$, for each $x \in \mathbb{N}$ and for each subset $A$ of $\mathbb{N}$, we can define the transition kernel from $\mathbb{N}$ to $\mathbb{N}$ :

$$
P_{i}(x, A)=\delta_{x} \star \mu_{i}(A) .
$$

The dynamic random walk on the dual of $S U(2)$ denoted by $\left(S_{n}\right)_{n \geq 0}$ is defined as the (inhomogeneous) Markov chain with state space $\mathbb{N}$ and transition kernel at time $n$ given by $P_{n}$. The probability to be in a subset $A$ of $\mathbb{N}$ at time $n$ when departing from state $x$ at time 0 is then given by

$$
P^{(n)}(x, A)=\delta_{x} \star \mu_{1} \star \ldots \star \mu_{n}(A)=\delta_{x} \star \mu^{(n)}(A)
$$

with the notation $\mu^{(n)}=\mu_{1} \star \ldots \star \mu_{n}$. 


\subsection{Limit theorems for dynamic random walks on the dual of $S U(2)$}

\subsubsection{A local limit theorem}

Assume that:

$\left(H_{1}\right): \forall i \geq 1, \mu_{i}$ is aperiodic. In particular, for every $\left.r \in\right] 0, \pi[$, there exists $\delta_{i}=\delta_{i}(r)$ such that

$$
\left|\hat{\mu}_{i}(\theta)\right| \leq 1-\delta_{i}, \quad \forall \theta \in[r, \pi]
$$

We will assume that

$$
n^{3 / 2} \prod_{i=1}^{n}\left(1-\delta_{i}\right)=o(1)
$$

$\left(H_{2}\right): \forall i \geq 1$

$$
\sum_{x \in \mathbb{N}} a_{x}^{(i)}(x+1)^{2}<+\infty
$$

$\left(H_{3}\right)$ : There exists a sequence of non negative reals $\left(A_{x}\right)_{x \in \mathbb{N}}$ such that $\sum_{x \in \mathbb{N}} A_{x}=$ $1, \sum_{x \in \mathbb{N}} A_{x}\left(x^{2}+2 x\right)<\infty$ and

$$
\lim _{n \rightarrow+\infty} \sum_{x \in \mathbb{N}}\left|\frac{1}{n} \sum_{i=1}^{n} a_{x}^{(i)}-A_{x}\right|(x+1)^{2}=0,
$$

$\left(H_{4}\right)$ :

$$
\sup _{i \geq 1}\left|\sum_{x \in \mathbb{N}}\left(a_{x}^{(i)}-A_{x}\right)(x+1)^{2}\right|<+\infty .
$$

Remark: If the union of the supports of the measures $\mu_{i}$ is reduced to a finite set $G$, assumptions $\left(H_{i}\right), i=2,3,4$ can be replaced by the following one:

$\left(H_{5}\right)$ : There exists a sequence of reals $\left(A_{x}\right)_{x \in \mathbb{N}}$ such that

$$
\lim _{n \rightarrow+\infty} \frac{1}{n} \sum_{i=1}^{n} a_{x}^{(i)}=A_{x}
$$

The support of the measure $\mu=\sum_{x \in \mathbb{N}} A_{x} \delta_{x}$ is evidently a subset of $G$.

Theorem 5.1 Under the assumptions $\left(H_{1}\right),\left(H_{2}\right),\left(H_{3}\right)$ and $\left(H_{4}\right)$,

$$
\lim _{n \rightarrow+\infty} \sup _{x \in \mathbb{N}}\left|2 \sqrt{\pi} n^{3 / 2} P^{(n)}(x, 0)-\frac{2 n}{(x+1) \sqrt{C}} e^{-\frac{(x+1)^{2}+1}{4 C n}} \sinh \left(\frac{x+1}{2 C n}\right)\right|=0
$$


where

$$
C=\frac{1}{6} \sum_{x \geq 0} A_{x}\left(x^{2}+2 x\right) .
$$

In particular: as $n \rightarrow \infty$,

$$
P^{(n)}(x, 0) \sim(2 \sqrt{\pi})^{-1} C^{-3 / 2} n^{-3 / 2} .
$$

\section{Proof:}

From formula (6),

$$
2 \sqrt{\pi} n^{3 / 2} P^{(n)}(x, 0)=\frac{4 n^{3 / 2}}{\sqrt{\pi}(x+1)} \int_{0}^{\pi} \widehat{\mu^{(n)}}(\theta) \sin ((x+1) \theta) \sin (\theta) d \theta .
$$

Using the change of variables: $\theta=\frac{\alpha}{\sqrt{n}}$, we get

$$
\begin{aligned}
2 \sqrt{\pi} n^{3 / 2} P^{(n)}(x, 0) & =\frac{4 n}{\sqrt{\pi}(x+1)} \int_{0}^{\pi \sqrt{n}} \widehat{\mu^{(n)}}\left(\frac{\alpha}{\sqrt{n}}\right) \sin \left((x+1) \frac{\alpha}{\sqrt{n}}\right) \sin \left(\frac{\alpha}{\sqrt{n}}\right) d \alpha \\
& =I_{0}(n)+I_{1}(n, A)+I_{2}(n, A)+I_{3}(n, A, r)+I_{4}(n A, r)
\end{aligned}
$$

where for some $A>0$ and $0<r<\pi$,

$$
\begin{aligned}
I_{0}(n) & =\frac{4 n}{\sqrt{\pi}(x+1)} \int_{0}^{+\infty} e^{-C \alpha^{2}} \sin \left((x+1) \frac{\alpha}{\sqrt{n}}\right) \sin \left(\frac{\alpha}{\sqrt{n}}\right) d \alpha \\
I_{1}(n, A) & =\frac{4 n}{\sqrt{\pi}(x+1)} \int_{0}^{A}\left[\widehat{\mu^{(n)}}\left(\frac{\alpha}{\sqrt{n}}\right)-e^{-C \alpha^{2}}\right] \sin \left((x+1) \frac{\alpha}{\sqrt{n}}\right) \sin \left(\frac{\alpha}{\sqrt{n}}\right) d \alpha \\
I_{2}(n, A) & =-\frac{4 n}{\sqrt{\pi}(x+1)} \int_{A}^{+\infty} e^{-C \alpha^{2}} \sin \left((x+1) \frac{\alpha}{\sqrt{n}}\right) \sin \left(\frac{\alpha}{\sqrt{n}}\right) d \alpha \\
I_{3}(n, A, r) & =\frac{4 n}{\sqrt{\pi}(x+1)} \int_{A}^{r \sqrt{n}} \widehat{\mu^{(n)}}\left(\frac{\alpha}{\sqrt{n}}\right) \sin \left((x+1) \frac{\alpha}{\sqrt{n}}\right) \sin \left(\frac{\alpha}{\sqrt{n}}\right) d \alpha \\
I_{4}(n, A, r) & =\frac{4 n}{\sqrt{\pi}(x+1)} \int_{r \sqrt{n}}^{\pi \sqrt{n}} \widehat{\mu^{(n)}}\left(\frac{\alpha}{\sqrt{n}}\right) \sin \left((x+1) \frac{\alpha}{\sqrt{n}}\right) \sin \left(\frac{\alpha}{\sqrt{n}}\right) d \alpha .
\end{aligned}
$$

Estimation of $I_{0}(n)$ :

From the well-known equality,

$$
\frac{1}{\sqrt{2 \pi}} \int_{\mathbb{R}} \cos (t x) e^{-x^{2} / 2} d x=e^{-t^{2} / 2}
$$

we deduce that

$$
\begin{aligned}
& \int_{0}^{+\infty} e^{-C \alpha^{2}} \sin \left((x+1) \frac{\alpha}{\sqrt{n}}\right) \sin \left(\frac{\alpha}{\sqrt{n}}\right) d \alpha \\
= & \frac{1}{2} \int_{0}^{+\infty} e^{-C \alpha^{2}} \cos \left(\frac{x \alpha}{\sqrt{n}}\right) d \alpha-\frac{1}{2} \int_{0}^{+\infty} e^{-C \alpha^{2}} \cos \left(\frac{(x+2) \alpha}{\sqrt{n}}\right) d \alpha \\
= & \frac{1}{2} \sqrt{\frac{\pi}{C}} e^{-\frac{(x+1)^{2}+1}{4 C n}} \sinh \left(\frac{x+1}{2 C n}\right) .
\end{aligned}
$$


Estimation of $I_{1}(n, A)$ :

For every $i \geq 1$, the Fourier transform of the distribution $\mu_{i}$ can be written as

$$
\hat{\mu_{i}}(\theta)=\frac{\phi_{i}(\theta)}{\sin (\theta)}
$$

with

$$
\phi_{i}(\theta)=\sum_{x \in \mathbb{N}} \frac{a_{x}^{(i)}}{(x+1)} \sin ((x+1) \theta) .
$$

In the same manner, the Fourier transform of the distribution $\mu:=\sum_{x \in \mathbb{N}} A_{x} \delta_{x}$ is given by

$$
\hat{\mu}(\theta):=\frac{\phi(\theta)}{\sin (\theta)}
$$

with

$$
\phi(\theta):=\sum_{x \in \mathbb{N}} \frac{A_{x}}{(x+1)} \sin ((x+1) \theta) .
$$

For every $x$, there exists $\eta=\eta(x) \in] 0,1[$ such that

$$
\sin (x)=x-\frac{x^{3}}{6}-\frac{x^{3}}{6}[\cos (\eta x)-1] .
$$

Then, from hypotheses $\left(H_{2}\right)$ and $\left(H_{3}\right)$, for every $i \geq 1$,

$$
\phi_{i}(\theta)=\theta-\frac{\theta^{3}}{6} \sum_{x \in \mathbb{N}} a_{x}^{(i)}(x+1)^{2}-\frac{\theta^{3}}{6} \sum_{x \in \mathbb{N}} a_{x}^{(i)}(x+1)^{2}\left[\cos \left(\eta_{x, \theta}(x+1) \theta\right)-1\right]
$$

and

$$
\phi(\theta)=\theta-\frac{\theta^{3}}{6} \sum_{x \in \mathbb{N}} A_{x}(x+1)^{2}-\frac{\theta^{3}}{6} \sum_{x \in \mathbb{N}} A_{x}(x+1)^{2}\left[\cos \left(\eta_{x, \theta}(x+1) \theta\right)-1\right]
$$

where $\eta_{x, \theta}$ are real numbers in $] 0,1[$ depending on $x$ and $\theta$.

Thus, for every $\alpha \in[0, A]$,

$$
\begin{aligned}
\left|\widehat{\mu^{(n)}}\left(\frac{\alpha}{\sqrt{n}}\right)-\hat{\mu}\left(\frac{\alpha}{\sqrt{n}}\right)^{n}\right| & =\left|\prod_{i=1}^{n} \hat{\mu}_{i}\left(\frac{\alpha}{\sqrt{n}}\right)-\hat{\mu}\left(\frac{\alpha}{\sqrt{n}}\right)^{n}\right| \\
& =\frac{1}{\left|\sin \left(\frac{\alpha}{\sqrt{n}}\right)\right|^{n}}\left|\prod_{i=1}^{n} \phi_{i}\left(\frac{\alpha}{\sqrt{n}}\right)-\phi\left(\frac{\alpha}{\sqrt{n}}\right)^{n}\right| \\
& =\frac{\left|\phi\left(\frac{\alpha}{\sqrt{n}}\right)\right|^{n}}{\left|\sin \left(\frac{\alpha}{\sqrt{n}}\right)\right|^{n}}\left|\exp \left(\sum_{i=1}^{n} \log \left(\frac{\phi_{i}\left(\frac{\alpha}{\sqrt{n}}\right)}{\phi\left(\frac{\alpha}{\sqrt{n}}\right)}\right)\right)-1\right|
\end{aligned}
$$


Using the following inequality,

$$
\left|e^{z}-1\right| \leq|z| e^{|z|}, \forall z \in \mathbb{C},
$$

we obtain that

$$
\left|\widehat{\mu^{(n)}}\left(\frac{\alpha}{\sqrt{n}}\right)-\hat{\mu}\left(\frac{\alpha}{\sqrt{n}}\right)^{n}\right| \leq \frac{\left|\phi\left(\frac{\alpha}{\sqrt{n}}\right)\right|^{n}}{\left|\sin \left(\frac{\alpha}{\sqrt{n}}\right)\right|^{n}}\left|\sum_{i=1}^{n} \log \left(\frac{\phi_{i}\left(\frac{\alpha}{\sqrt{n}}\right)}{\phi\left(\frac{\alpha}{\sqrt{n}}\right)}\right)\right| \exp \left|\sum_{i=1}^{n} \log \left(\frac{\phi_{i}\left(\frac{\alpha}{\sqrt{n}}\right)}{\phi\left(\frac{\alpha}{\sqrt{n}}\right)}\right)\right|
$$

Now, thanks to (11),

$$
\lim _{n \rightarrow \infty} \sup _{\alpha \in[0, A]}\left|\frac{\phi\left(\frac{\alpha}{\sqrt{n}}\right)^{n}}{\sin \left(\frac{\alpha}{\sqrt{n}}\right)^{n}}-\exp \left(-C \alpha^{2}\right)\right|=0 .
$$

It remains to prove that

$$
\lim _{n \rightarrow \infty} \sup _{\alpha \in[0, A]}\left|\sum_{i=1}^{n} \log \left(\frac{\phi_{i}\left(\frac{\alpha}{\sqrt{n}}\right)}{\phi\left(\frac{\alpha}{\sqrt{n}}\right)}\right)\right|=0 .
$$

For $n$ large enough, thanks to $\left(H_{4}\right),(10)$ and (11),

$$
\begin{aligned}
\sum_{i=1}^{n} \log \left(\frac{\phi_{i}\left(\frac{\alpha}{\sqrt{n}}\right)}{\phi\left(\frac{\alpha}{\sqrt{n}}\right)}\right) & =\sum_{i=1}^{n} \log \left(1+\frac{\phi_{i}\left(\frac{\alpha}{\sqrt{n}}\right)-\phi\left(\frac{\alpha}{\sqrt{n}}\right)}{\phi\left(\frac{\alpha}{\sqrt{n}}\right)}\right) \\
& =-\frac{\alpha^{2}}{6 n} \sum_{i=1}^{n} \sum_{x \in \mathbb{N}}\left[a_{x}^{(i)}-A_{x}\right](x+1)^{2} \\
& -\frac{\alpha^{2}}{6 n} \sum_{i=1}^{n} \sum_{x \in \mathbb{N}}\left[a_{x}^{(i)}-A_{x}\right](x+1)^{2}\left[\cos \left(\eta_{x, \theta}(x+1) \theta\right)-1\right]+o(1) \\
& =o(1)
\end{aligned}
$$

using hypothesis $\left(H_{3}\right)$.

From the inequality: $|\sin (x)| \leq x$ for $x \geq 0$, we get that

$$
\left|I_{1}(n, A)\right| \leq \frac{4 A^{3}}{3 \sqrt{\pi}} \sup _{0 \leq \alpha \leq A}\left|\widehat{\mu^{(n)}}\left(\frac{\alpha}{\sqrt{n}}\right)-\exp \left(-C \alpha^{2}\right)\right|=o(1)
$$

uniformly in $x$.

Estimation of $I_{2}(n, A)$ :

Since $|\sin (x)| \leq x$ for $x \geq 0$, we have

$$
\left|I_{2}(n, A)\right| \leq \frac{4}{\sqrt{\pi}} \int_{A}^{+\infty} \alpha^{2} \exp \left(-C \alpha^{2}\right) d \alpha \rightarrow 0
$$


when $A$ goes to infinity, uniformly in $x$.

Estimation of $I_{3}(n, A, r)$ :

$$
I_{3}(n, A, r)=\frac{4 n}{\sqrt{\pi}(x+1)} \int_{A}^{r \sqrt{n}} \widehat{\mu^{(n)}}\left(\frac{\alpha}{\sqrt{n}}\right) \sin \left((x+1) \frac{\alpha}{\sqrt{n}}\right) \sin \left(\frac{\alpha}{\sqrt{n}}\right) d \alpha
$$

We can choose $r$ small enough to have

$$
\left|\widehat{\mu^{(n)}}\left(\frac{\alpha}{\sqrt{n}}\right)\right| \leq \exp \left(-\frac{\alpha^{2}}{6 n} \sum_{i=1}^{n} \sum_{x \in \mathbb{N}} a_{x}^{(i)}\left(x^{2}+2 x\right)\right)
$$

Then, from hypothesis $\left(H_{3}\right)$, there exists a constant $C^{\prime}$ strictly positive such that

$$
\left|\widehat{\mu^{(n)}}\left(\frac{\alpha}{\sqrt{n}}\right)\right| \leq \exp \left(-C^{\prime} \alpha^{2}\right)
$$

So,

$$
\left|I_{3}(n, A, r)\right| \leq \frac{4}{\sqrt{\pi}} \int_{A}^{+\infty} \alpha^{2} \exp \left(-C^{\prime} \alpha^{2}\right) d \alpha \rightarrow 0
$$

when $A$ goes to infinity, uniformly in $x$.

Estimation of $I_{4}(n, A, r)$ :

$$
\begin{aligned}
I_{4}(n, A, r) & =\frac{4 n}{\sqrt{\pi}(x+1)} \int_{r \sqrt{n}}^{\pi \sqrt{n}} \widehat{\mu^{(n)}}\left(\frac{\alpha}{\sqrt{n}}\right) \sin \left((x+1) \frac{\alpha}{\sqrt{n}}\right) \sin \left(\frac{\alpha}{\sqrt{n}}\right) d \alpha \\
& =\frac{4 n^{3 / 2}}{\sqrt{\pi}(x+1)} \int_{r}^{\pi} \widehat{\mu^{(n)}}(\theta) \sin ((x+1) \theta) \sin (\theta) d \theta
\end{aligned}
$$

For every $i \geq 1$, the probability measure $\mu_{i}$ being aperiodic, there exists $\delta_{i}=\delta_{i}(r)>0$ such that

$$
\left|\hat{\mu}_{i}(\theta)\right| \leq 1-\delta_{i}, \quad \forall \theta \in[r, \pi]
$$

SO

$$
\left|I_{4}(n, A, r)\right| \leq \frac{4 \sqrt{\pi} n^{3 / 2}}{(x+1)} \prod_{i=1}^{n}\left(1-\delta_{i}\right)=o(1)
$$

uniformly in $x$. 


\subsubsection{Recurrence and transience}

The question of recurrence-transience is natural and can be deduced from a slightly modification of the previous local limit theorem.

Definition 5.1 The dynamic random walk on the dual of $S U(2)$ is transient if and only if for every point $x \in \mathbb{N}, y \in \mathbb{N}$, the walk starting from $x$ visits $y$ only a finite number of times. It is equivalent to say that, for every point $x \in \mathbb{N}, y \in \mathbb{N}$, the potential kernel defined by

$$
G(x, y)=\sum_{n \geq 0} P^{(n)}(x, y)
$$

is finite.

Under the assumptions $\left(H_{1}\right),\left(H_{2}\right),\left(H_{3}\right)$ and $\left(H_{4}\right)$,

$\lim _{n \rightarrow+\infty} \sup _{x \in \mathbb{N}}\left|2 \sqrt{\pi} n^{3 / 2} P^{(n)}(x, y)-\frac{2 n(y+1)}{\sqrt{C}(x+1)} e^{-\frac{(x+1)^{2}+(y+1)^{2}}{4 C n}} \sinh \left(\frac{(x+1)(y+1)}{2 C n}\right)\right|=0$

where

$$
C=\frac{1}{6} \sum_{x \geq 0} A_{x}\left(x^{2}+2 x\right) .
$$

In particular: for every point $x \in \mathbb{N}, y \in \mathbb{N}$, as $n \rightarrow \infty$,

$$
P^{(n)}(x, y) \sim(y+1)^{2}(2 \sqrt{\pi})^{-1} C^{-3 / 2} n^{-3 / 2} .
$$

From this result, we easily deduce the following theorem

Theorem 5.2 Under assumptions $\left(H_{1}\right),\left(H_{2}\right),\left(H_{3}\right)$ and $\left(H_{4}\right)$, the dynamic random walk on the dual of $S U(2)$ is transient.

\subsubsection{A central limit theorem}

From the local limit theorem established in Section 5.2.1., we deduce a central limit theorem for the dynamic random walk on the dual of $S U(2)$.

Theorem 5.3 Under the assumptions $\left(H_{1}\right),\left(H_{2}\right),\left(H_{3}\right)$ and $\left(H_{4}\right)$, the sequence $Y_{n}=\frac{S_{n}}{\sqrt{2 C n}}$ where

$$
C=\frac{1}{6} \sum_{x \geq 0} A_{x}\left(x^{2}+2 x\right) .
$$


converges in distribution, as $n \rightarrow+\infty$, to the random variable whose the distribution density is

$$
\sqrt{\frac{2}{\pi}} x^{2} \exp \left(-\frac{x^{2}}{2}\right)
$$

\section{Proof:}

For $0 \leq a \leq b$, we have the relation

$$
\begin{aligned}
\mathbb{P}\left(a \leq Y_{n} \leq b\right) & =\mathbb{P}\left(a \sqrt{2 C n} \leq S_{n} \leq b \sqrt{2 C n}\right) \\
& =\sum_{a \sqrt{2 C n} \leq x \leq b \sqrt{2 C n} ; x \in \mathbb{N}} \frac{2(x+1)}{\pi} \int_{0}^{\pi} \widehat{\mu^{(n)}}(\theta) \sin ((x+1) \theta) \sin (\theta) d \theta .
\end{aligned}
$$

Using the changes of variables: $y=\frac{(x+1)}{\sqrt{n}}$ and $\theta=\frac{\alpha}{\sqrt{n}}$, we get

$$
\mathbb{P}\left(a \leq Y_{n} \leq b\right)=\sum_{a \sqrt{2 C n}+1 \leq y \sqrt{n} \leq b \sqrt{2 C n}+1 ; y \sqrt{n} \in \mathbb{N}} \frac{2 y}{\pi} \int_{0}^{\pi \sqrt{n}} \widehat{\mu^{(n)}}\left(\frac{\alpha}{\sqrt{n}}\right) \sin (y \alpha) \sin \left(\frac{\alpha}{\sqrt{n}}\right) d \alpha
$$

Now,

$$
\lim _{n \rightarrow+\infty} \frac{1}{\sqrt{n}} \sum_{a \sqrt{2 C n}+1 \leq y \sqrt{n} \leq b \sqrt{2 C n}+1 ; y \sqrt{n} \in \mathbb{N}} y \sin (y \alpha)=\int_{a \sqrt{2 C}}^{b \sqrt{2 C}} y \sin (y \alpha) d y
$$

and

$$
\lim _{n \rightarrow+\infty} \widehat{\mu^{(n)}}\left(\frac{\alpha}{\sqrt{n}}\right)=\exp \left(-C \alpha^{2}\right)
$$

Thus,

$$
\lim _{n \rightarrow \infty} \mathbb{P}\left(a \leq Y_{n} \leq b\right)=\frac{2}{\pi} \int_{a \sqrt{2 C}}^{b \sqrt{2 C}} y\left[\int_{0}^{\infty} \alpha \sin (y \alpha) \exp \left(-C \alpha^{2}\right) d \alpha\right] d y .
$$

Integrating by parts and using formula (9), we get

$$
\int_{0}^{\infty} \alpha \sin (y \alpha) \exp \left(-C \alpha^{2}\right) d \alpha=\frac{y \sqrt{\pi}}{4 C^{3 / 2}} \exp \left(-y^{2} /(4 C)\right) .
$$

Thus,

$$
\lim _{n \rightarrow \infty} \mathbb{P}\left(a \leq Y_{n} \leq b\right)=\sqrt{\frac{2}{\pi}} \int_{a}^{b} y^{2} \exp \left(-\frac{y^{2}}{2}\right) d y
$$

Example: Let $S=(E, \mathcal{A}, \mu, T)$ be a dynamical system where $(E, \mathcal{A}, \mu)$ is a probability space and $T$ is a measure-preserving transformation defined on $E$. 
For each $e \in E$, let $a_{x}^{(i)}=a_{x}\left(T^{i} e\right)$ where $a_{x}$ are positive functions such that $\sum_{x \geq 0} a_{x}^{(i)}(e)=1$. A particular example is $a_{x}=0$ if $x \neq 1,2$ and $a_{1}(e)=f(e)$ and $a_{2}(e)=1-f(e)$ where $f$ is a measurable function defined on $E$ with values in $] 0,1[$. This is a model similar to the dynamic random walks considered in $[12,13,14,15,16,17,18]$. Under assumption of uniform ergodicity of the dynamical system, assumptions $\left(H_{i}\right), i=1, \ldots, 4$ are clearly satisfied and in that case, Theorems 5.1, 5.2 and 5.3 hold with the constant $C=\frac{1}{3}\left[4-\frac{5}{2} \int_{E} f d \mu\right]>0$.

\subsubsection{A weak law of large numbers}

From the central limit theorem (Theorem 5.3), we deduce the

Theorem 5.4 Under the assumptions $\left(H_{1}\right),\left(H_{2}\right),\left(H_{3}\right)$ and $\left(H_{4}\right)$, for every $\epsilon>0$, the sequence of random variables $\left(S_{n} / n^{1 / 2+\epsilon}\right)_{n \geq 1}$ converges in probability to 0 , as $n$ goes to infinity.

\section{Remark:}

$\epsilon=1 / 2$ provides the traditional law of large numbers. A strong law of large numbers should hold using the method developed in Section III of [11]. Details are omitted. This will be the object of further investigation.

\subsubsection{A large deviations principle}

In the previous section, $S_{n} / n$ was proved to converge in probability to 0 under convenient assumptions, so the sequence of random variables $\left(S_{n} / n\right)_{n}$ is a good candidate for a large deviations principle. Let us first recall what we mean by Large Deviations Principle: Let $\Gamma$ be a Polish space endowed with the Borel $\sigma$-field $\mathcal{B}(\Gamma)$. A good rate function is a lower semi-continuous function $\Lambda^{*}: \Gamma \rightarrow[0, \infty]$ with compact level sets $\left\{x ; \Lambda^{*}(x) \leq \alpha\right\}, \alpha \in[0, \infty[$. Let $v=\left(v_{n}\right)_{n} \uparrow \infty$ be an increasing sequence of positive reals. A sequence of random variables $\left(Y_{n}\right)_{n}$ with values in $\Gamma$ defined on a probability space $(\Omega, \mathcal{F}, \mathbb{P})$ is said to satisfy a Large Deviations Principle (LDP) with speed $v=\left(v_{n}\right)_{n}$ and the good rate function $\Lambda^{*}$ if for every Borel set $B \in \mathcal{B}(\Gamma)$,

$$
\begin{aligned}
-\inf _{x \in B^{o}} \Lambda^{*}(x) & \leq \liminf _{n} \frac{1}{v_{n}} \log \mathbb{P}\left(Y_{n} \in B\right) \\
& \leq \limsup _{n} \frac{1}{v_{n}} \log \mathbb{P}\left(Y_{n} \in B\right) \leq-\inf _{x \in \bar{B}} \Lambda^{*}(x) .
\end{aligned}
$$


When the upper inequality holds, $\left(Y_{n}\right)_{n}$ is said to satisfy an upper Large Deviations Principle. Let us consider now a sequence of probability measures $\left(\mu_{i}\right)_{i \geq 1}$ where $\mu_{i}=\sum_{x \in \mathbb{N}} a_{x}^{(i)} \delta_{x}$ on $\mathbb{N}$ (remember that $a_{x}^{(i)}$ are coefficients which are non negative and $\left.\sum_{x \in \mathbb{N}} a_{x}^{(i)}=1\right)$. We denote by $\tilde{\mu}_{i}$ the Laplace transform of the measure $\mu_{i}$.

We make the following assumption:

$(H)$ : the function $\Gamma$ defined by

$$
\forall t \in \mathbb{R}^{+}, \quad \Gamma(t):=\limsup _{n \rightarrow+\infty} \frac{1}{n} \sum_{i=1}^{n} \log \tilde{\mu}_{i}(t)
$$

is finite in a neighbourhood of 0 .

Theorem 5.5 Under Assumption (H), the sequence of random variables $\left(S_{n} / n\right)_{n \geq 1}$ satisfies an upper Large Deviations Principle: for every $y \in \mathbb{R}^{+, *}$,

$$
\limsup _{n \rightarrow+\infty} \frac{1}{n} \log \mathbb{P}\left(\frac{S_{n}}{n} \geq y\right) \leq-\Lambda^{*}(y)
$$

where

$$
\Lambda^{*}(y)=\sup _{t ; \Gamma(t)<\infty}\{t y-\Gamma(t)\}
$$

Assume that the function $\Lambda^{*}$ is strictly positive on $\mathbb{R}^{+, *}$, then the sequence of random variables $\left(S_{n} / n\right)_{n \geq 1}$ converges almost surely to 0 as $n$ goes to infinity.

\section{Proof:}

Since the function $x \rightarrow \frac{\sinh [(x+1) t]}{(x+1) \sinh (t)}$ is increasing on $\mathbb{R}^{+}$, using the Markov inequality, we get

$$
\begin{aligned}
\mathbb{P}\left(\frac{S_{n}}{n} \geq y\right) & =\mathbb{P}\left(\frac{\sinh \left[\left(S_{n}+1\right) t\right]}{\left(S_{n}+1\right) \sinh (t)} \geq \frac{\sinh [(n y+1) t]}{(n y+1) \sinh (t)}\right) \\
& \leq \mathbb{E}\left(\frac{\sinh \left[\left(S_{n}+1\right) t\right]}{\left(S_{n}+1\right) \sinh (t)}\right) \frac{(n y+1) \sinh (t)}{\sinh [(n y+1) t]}
\end{aligned}
$$

Now, from the definition of the Laplace transform and formula (8),

$$
\begin{aligned}
\mathbb{E}\left(\frac{\sinh \left[\left(S_{n}+1\right) t\right]}{\left(S_{n}+1\right) \sinh (t)}\right) & =\sum_{x \in \mathbb{N}} \psi_{x}(t) \delta_{0} \star \mu^{(n)}(x) \\
& =\left(\delta_{0} \approx \mu^{(n)}\right)(t) \\
& =\prod_{i=1}^{n} \tilde{\mu}_{i}(t)
\end{aligned}
$$


Moreover

$$
\frac{\sinh (t)}{\sinh [(n y+1) t]} \leq e^{-n y t}
$$

Hence,

$$
\frac{1}{n} \log \mathbb{P}\left(\frac{S_{n}}{n} \geq y\right) \leq-t y+\frac{1}{n} \log (n y+1)+\frac{1}{n} \sum_{i=1}^{n} \log \left(\tilde{\mu}_{i}(t)\right)
$$

Then, using assumption $(\mathrm{H})$,

$$
\begin{aligned}
\limsup _{n \rightarrow+\infty} \frac{1}{n} \log \mathbb{P}\left(\frac{S_{n}}{n} \geq y\right) & \leq-t y+\limsup _{n \rightarrow+\infty} \frac{1}{n} \sum_{i=1}^{n} \log \left(\tilde{\mu}_{i}(t)\right) \\
& \leq-t y+\Gamma(t) .
\end{aligned}
$$

Taking the infimum in the right hand side over all $t>0$, we get the result.

Let $\epsilon>0$. For $n$ large enough,

$$
\mathbb{P}\left(\frac{S_{n}}{n} \geq \epsilon\right) \leq e^{-n \Lambda^{*}(\epsilon) / 2}
$$

Since the series $\sum_{n} e^{-n \alpha}$ is finite for any $\alpha>0$, from Borel-Cantelli lemma, we deduce that $S_{n} / n$ converges almost surely to 0 as $n$ goes to infinity.

Example: Consider the example from Section 5.1.3 that is a dynamical system $(E, \mathcal{A}, \mu, T)$ where $(E, \mathcal{A}, \mu)$ is a probability space and $T$ is a transformation on $E$. Let $e \in E$ a fixed point, and choose $a_{x}^{(i)}=a_{x}\left(T^{i} e\right)$ where $a_{x}=0$ if $x \neq 1,2, a_{1}(e)=f(e)$ and $a_{2}(e)=1-f(e)$ where $f$ is a measurable function defined on $E$ with values in ]0,1[. Then, for every $i \geq 1$, the Laplace transform of the measure $\mu_{i}=\sum_{x} a_{x}^{(i)} \delta_{x}$ is given by

$$
\tilde{\mu}_{i}(t)=\cosh (t) f\left(T^{i} e\right)+\left(1-f\left(T^{i} e\right)\right) \frac{\sinh (3 t)}{3 \sinh (t)} .
$$

When the dynamical system is uniformly ergodic (e.g. $T$ is an irrational rotation on the torus), assumption $(\mathrm{H})$ is clearly satisfied with the function

$$
\Gamma(t)=\int_{E} \log \left[\cosh (t) f(x)+(1-f(x)) \frac{\sinh (3 t)}{3 \sinh (t)}\right] d \mu(t) .
$$

It is worth remarking that using Jensen's inequality, the function $\Gamma$ is less than or equal to $\log (\tilde{\mu}(t))$ where $\mu$ is the probability measure $\int_{E} f d \mu \delta_{1}+(1-$ $\left.\int_{E} f d \mu\right) \delta_{2}$. Consequently,

$$
-\sup \{t x-\Gamma(t)\} \leq-\sup \{t x-\log \tilde{\mu}(t)\} .
$$


Assumption $(H)$ is weaker than assuming that the average of the $\tilde{\mu}_{i}$ 's converges to $\tilde{\mu}$. Take for instance the irrational rotation on the one-dimensional torus and $f=\mathbf{1}_{[0,1 / 2]}$, then

$$
\Gamma(t)=\frac{1}{2}\left[\log (\cosh (t))+\log \left(\frac{\sinh (3 t)}{3 \sinh (t)}\right)\right]
$$

and

$$
\log (\tilde{\mu}(t))=\log \left[\frac{1}{2} \cosh (t)+\frac{1}{2} \frac{\sinh (3 t)}{3 \sinh (t)}\right] .
$$

We recover the fact that the dynamic random walk is more concentrated around the origin as the random walk with stationary increments, a phenomenon already encountered in [8].

\section{Concluding remarks}

As stated in the introduction, this paper is a first attempt in extending the theory of dynamic random walks developed by the first author to noncommutative algebraic structures.

\section{References}

[1] AcCARdi L., Frigerio A. ANd Lewis, J.T. Quantum stochastic processes. Publ. R.I.M.S, Kyoto (1982), 97-133.

[2] Biane, P. Marches de Bernoulli quantiques. Lect. Notes Math. 1426 (1990), 329-344, Springer Verlag.

[3] Biane, P. Some properties of quantum Bernoulli random walks. Proceedings of Quantum Probabilities 6 (1989), 193-203.

[4] Biane, P. Quantum random walk on the dual of $S U(n)$. Proba. Th. Rel. Fields (1991), 89, 117-129,Springer Verlag.

[5] Biane, P. Calcul stochastique non-commutatif. Lect. Notes Math. 1608 (1994), 1-93, Springer Verlag.

[6] Biane, P. Théorème de Ney-Spitzer sur le dual de SU(2). Transactions of the American Mathematical Society (1994), 345, 1, 179-194. 
[7] DixmieR, J. Les $C^{*}$-algèbres et leurs représentations. (1964), GauthierVillars.

[8] Dombry, C., Guillotin-Plantard, N., Pinçon, B. and Schott, R. Data structures with dynamical random transitions. Random Structures and Algorithms (in press).

[9] Eymard, P. And Roynette, B. Marches aléatoires sur le dual de SU(2). Lect. Notes Math. 497 (1975), 108-152, Springer-Verlag.

[10] Gallardo, L. Une transformation de Cramér sur le dual de SU(2). Ann. Sci. Univ. Clermont-Ferrand II Math. (1982), No 20, 102-106.

[11] Gallardo, L. AND Ries V. La loi des grands nombres pour les marches aléatoires sur le dual de SU(2). Studia mathematica (1979) , Vol. 66, 133-148.

[12] Guillotin, N. Asymptotics of a dynamic random walk in a random scenery I. A law of large numbers. Annales de l'Institut Henri Poincaré - Probabilités et Statistiques (2000), Vol. 36, No 2, 127-151.

[13] Guillotin, N. Asymptotics of a dynamic random walk in a random scenery II. A functional limit theorem. Markov Processes and Related Fields (1999), Vol. 5, No 2, 201-218.

[14] Guillotin-Plantard, N. Dynamic $\mathbb{Z}^{d}$-random walks in a random scenery: a strong law of large numbers. J. Theoret. Probab. (2001), Vol. 14, No 1, 241-260.

[15] Guillotin-Plantard, N. And Schott, R. Distributed algorithms with dynamical random transitions. Random Structures and Algorithms (2002), 21, 3-4, 376-395.

[16] Guillotin-Plantard, N. And Schott, R. Dynamic random walks: Theory and applications . 200 pages, (book in press).

[17] Guillotin-Plantard, N. And Schott, R. Dynamic random walks on Heisenberg groups. Journal of Theoretical Probability (in press).

[18] Guillotin-Plantard, N., Schott, R. and Schurz, H. Dynamic $(B, S)$-markets. Prépublication Institut E. Cartan No 17, 2004.

[19] Guivarc'H, Y.; Keane, M.; Roynette, B. Marches aléatoires sur les groupes de Lie. Lect. Notes Math. 624 (1977) Springer-Verlag. 
[20] Parthasarathy, K.R. A generalized Biane's process. Lect. Notes Math. 1426 (1990), 345-348, Springer Verlag. 